\title{
THE EQUIVARIANT COHOMOLOGY OF WEIGHTED FLAG ORBIFOLDS
}

\author{
HANIYA AZAM, SHAHEEN NAZIR, AND MUHAMMAD IMRAN QURESHI
}

\begin{abstract}
We describe the torus-equivariant cohomology of weighted partial flag orbifolds $\mathrm{w} \Sigma$ of type $A$. We establish counterparts of several results known for the partial flag variety that collectively constitute what we refer to as "Schubert Calculus on $\mathrm{w} \Sigma$ ". For the weighed Schubert classes in $\mathrm{w} \Sigma$, we give the Chevalley's formula. In addition, we define the weighted analogue of double Schubert polynomials and give the corresponding Chevalley-Monk's formula.
\end{abstract}

\section{INTRODUCTION}

A flag variety is the quotient of a reductive Lie group $G$ by a unique parabolic subgroup $P$ (up to conjugation) that is,

$$
\Sigma=G / P
$$

Alternatively, it can be described as a projective subvariety of the projectivization of some irreducible $G$-representation. The notion of a weighted flag variety (WFV) $\mathrm{w} \Sigma$, is the weighted projective analogue of the flag variety, introduced by Grojnowski, Corti and Reid [CR02]. Any WFV is locally covered by open sets which are quotients of affine spaces by finite groups, giving it the structure of an orbifold. Thus we use the terms variety and orbifold interchangeably throughout the paper. Ever since their introduction, various types of WFVs have been used to serve as ambient varieties to construct some interesting classes of polarized orbifolds such as canonical Calabi-Yau 3-folds, log-terminal Fano 3folds and canonical 3-folds etc. (see[CR02, QS11, Qur15, QS12, BKZ14, Qur17a, Qur17b). On the topological side, the equivariant cohomology of weighted Grassmannians has been computed by Abe and Matsumura in AM15a.

An important early formal reference on the topology of certain homogeneous spaces, in particular the flag manifolds, dates back to 1934 by Ehresmann Ehr34. Borel in his fundamental work on transformation groups $\left[\mathrm{BBF}^{+} 60\right]$ defined what is now called the equivariant cohomology of a space with some group action defined on it. In his work Borel applied spectral sequence to the topology of Lie groups and their classifying spaces showing that they degenerate to equivariant cohomology of homogeneous spaces. In [CS73], Chang and Skjelbred proposed the idea of restricting attention to one-dimensional orbits for calculating equivariant cohomology. Berligne and Vergne $\left[\mathrm{BV}^{+} 83\right]$ gave the localization theorem in the context of moment map, a point of view also adopted by Attiyah and Bott in [AB94]. An integration of these ideas in terms of the equivariant cohomology ring of

Key words and phrases. Weighted flag varieties, equivariant cohomology, Schubert classes, double Schubert polynomials. 
'equivariantly formal spaces' (for instance, homogeneous spaces) was given by Goresky, Kottwitz and MacPherson in [GKM97. They defined the 'equivariantly formal spaces' as spaces whose $G$-equivariant cohomology can be computed by restricting attention to fixed points and one-dimensional orbits of the maximal torus inside $G$. The first computation of equivariant cohomology for the complete flag variety was given by Arabia in [Alb86. Afterwards, many people used [GKM97] to describe equivariant cohomology of different spaces. The GKM description of the equivariant cohomology ring of general homogenous spaces has been given by Guillemin, Holm and Zara in [GHZ06] and for partial flag varieties of type $A$, by Tymoczko in Tym09.

We compute the rational torus-equivariant cohomology of weighted flag orbifolds $\mathrm{w} \Sigma$ of type $A$, generalizing [AM15a to the case of weighted partial flag varieties. We generalize some known results for partial flag varieties to WFVs, which includes providing the GKM description of the cohomology ring, a Chevalley's formula for $\mathrm{w} \Sigma$ and the corresponding Chevalley-Monk's formula in terms of weighted Schubert polynomials defined later.

In $\$ 2$ we lay the representation theoretic foundations needed in the rest of the paper. We recall the precise relation between a parabolic subgroup $P$ and an irreducible representation $V_{\chi}$ of $G$, where $\chi$ is the highest weight. For a given choice of torus $T$ inside $G$, we explicitly describe the $T$-invariant basis of the highest weight representation $V_{\chi}$ using Deyrut's construction of Schur modules. This construction allows us to explicitly compute the weights of the representation $V_{\chi}$.

In $\$ 3$ we recall the Bruhat order on Schubert cells and describe the open charts and weighted cell decomposition of $\mathrm{w} \Sigma$ using a $G$-equivariant Plücker type embedding of $\mathrm{w} \Sigma$. These open charts are all isomorphic to a quotient of a complex Euclidean space by some finite cyclic group. We give an explicit formula to compute the ranks of singular rational cohomology groups of these WFVs using Borel-Moore homology. As a consequence of equivariant formality we show that the equivariant cohomology ring of $\mathrm{w} \Sigma$ admits a basis over the equivariant cohomology of torus-fixed points.

Theorem 1.1. There is an $H^{*}\left(B T_{\mathrm{w}}\right)$-module isomorphism

$$
H_{T_{\mathrm{w}}}^{*}(\mathrm{w} \Sigma) \cong H^{*}\left(B T_{\mathrm{w}}\right) \otimes_{\mathbb{Q}} H^{*}(\mathrm{w} \Sigma),
$$

where $H_{T_{\mathrm{w}}}^{*}($.$) is the T_{\mathrm{w}}$-equivariant cohomology and $B T_{\mathrm{w}}$ is the classifying space of $T_{\mathrm{w}}(\cong$ $T)$. In fact, $H_{T_{\mathrm{w}}}^{*}(\mathrm{w} \Sigma)$ is a free module.

Apart from giving the module structure we also describe the equivariant cohomology ring of $\mathrm{w} \Sigma$ following Kirwan Kir84. We use the explicit description of $\mathrm{w} \Sigma$ as the quotient of a compact real symplectic submanifold of the punctured affine cone $a \Sigma^{\times}$by a Hamiltonian action of the real torus inside $\mathbb{C}^{\times}$.

Theorem 1.2. There exists a compact real symplectic submanifold $M$ of the punctured affine cone $\mathrm{a}^{\times}$such that

$$
H_{T_{\mathrm{w}}}^{*}(\mathrm{w} \Sigma) \cong H_{S_{T_{\mathrm{w}}}}^{*}\left(M / S^{1}\right) .
$$

In $\$ 4$ we define Schubert classes both in the weighted flag orbifold $\mathrm{w} \Sigma$ and the punctured affine cone $a \Sigma^{\times}$, using the structure of a $\Sigma^{\times}$as a quasi-projective variety. These classes are 
defined as pullbacks of torus-equivariant Schubert classes in the flag variety $\Sigma$. We also give the combinatorial description, commonly known as GKM description of equivariant cohomology rings $H_{K}^{*}\left(\mathrm{a} \Sigma^{\times}\right)$and $H_{T_{\mathrm{w}}}^{*}(\mathrm{w} \Sigma)$. This is done by describing the image of the injection

$$
H_{T}^{*}(\Sigma) \hookrightarrow \bigoplus_{\sigma \in W^{P}} H_{T}^{*}\left(\left[e_{\sigma}\right]\right)
$$

where $e_{\sigma}$ is a torus-fixed point in $\Sigma$ and $W^{P}$ is defined explicitly in Section 3.1 .

In $\$ 5$ using these GKM descriptions we give Chevalley's formula (Theorem 5.1) for the weighted flag orbifold $\mathrm{w} \Sigma$ by generalizing the classical formula of Kostant and Kumar [KK86]. We define weighted Schubert polynomials $\mathrm{w}_{\sigma}(x)$ for a weighted flag orbifold $\mathrm{w} \Sigma$, which generalize the known double Schubert polynomials defined by Lascoux and Schutzenberger. The weighted Schubert polynomials correspond to weighted equivariant cohomology classes of the WFVs. We also give the weighted Chevalley-Monk's formula (Theorem 5.10 in terms of weighted Schubert polynomials.

\section{IRREDUCiBle REPRESEntations of $\operatorname{GL}(n, \mathbb{C})$}

2.1. Background. Let $G=\mathrm{GL}(n, \mathbb{C}), B$ be the Borel group of upper triangular matrices, $P$ be a parabolic subgroup of $G$, and $T$ be the maximal torus of diagonal matrices with associated Lie algebras $\mathfrak{t} \subset \mathfrak{b} \subset \mathfrak{p} \subset \mathfrak{g l}_{n}(\mathbb{C})$. Let

$$
\Xi(T)=\operatorname{Hom}\left(T, \mathbb{C}^{\times}\right)=\left\langle L_{1}, L_{2}, \ldots, L_{n}\right\rangle
$$

be the weight lattice. For each $k=1, \ldots, n-1$, the $G$-representation $\wedge^{k} \mathbb{C}^{n}$ is called fundamental representation of $G$, having the highest weight

$$
\omega_{k}=\sum_{i=1}^{k} L_{i} \in \Xi(T), \quad 1 \leq k \leq n-1 .
$$

Let $\Delta$ be the root system of the Lie algebra $\mathfrak{g}$ corresponding to $G$. Let

$$
\Delta_{s}:=\left\{\alpha_{i}=L_{i}-L_{i+1}, 1 \leq i \leq n-1\right\}
$$

be the set of simple roots of $\mathfrak{g}$. The Weyl group $W$ of $\Delta$ is generated by reflections in the hyperplane perpendicular to each simple root $\alpha_{i}$, i.e.,

$$
W=\left\langle s_{\alpha_{i}}: \alpha_{i} \in \Delta_{s}\right\rangle \cong S_{n},
$$

where isomorphism with $S_{n}$ is obtained by mapping each simple root $\alpha_{i}$ to the simple transposition $s_{i}$. There is a one-one correspondence between parabolic subgroups and subsets of $\Delta_{s}$ (see [FH13, Section 23.3]). Consider the flag variety defined by $P$,

$$
\Sigma=G / P:=\left\{F_{\bullet}: 0=V_{0} \subset V_{1} \subset \cdots \subset V_{r} \subset V_{r+1}=\mathbb{C}^{n} \mid \operatorname{dim}\left(V_{i}\right)=d_{i}\right\},
$$

where $P$ is the stabilizer of the $G$-action on each flag in $\Sigma$. The parabolic subgroup $P$ corresponds to the subset $J=\left\{\alpha_{i}: i \notin\left\{d_{1}, \ldots, d_{r}\right\}\right\}$ of $\Delta_{s}$ and $W_{P}=<s_{i}: \alpha_{i} \in J>$ denotes the corresponding subgroup of the Weyl group $W$. The dimension of $\Sigma$ by [Bri05] equals

$$
\operatorname{dim}(\Sigma)=\sum_{i=1}^{r} d_{i}\left(d_{i+1}-d_{i}\right), \text { where } d_{r+1}=n .
$$


The flag variety $\Sigma$ is a projective subvariety of $\mathbb{P} V_{\chi}$, where $V_{\chi}$ is an irreducible $G$ representation with highest weight

$$
\chi=\sum_{i=1}^{r} \omega_{d_{i}}=r\left(L_{1}+\cdots+L_{d_{1}}\right)+(r-1)\left(L_{d_{1}+1}+\cdots+L_{d_{2}}\right)+\cdots+\left(L_{d_{r-1}+1}+\cdots+L_{d_{r}}\right) .
$$

Following [Ful97], the Young diagram associated to $\chi$ is of length $r$ and has type

$$
\chi=(\underbrace{r, \ldots, r}_{d_{1}}, \underbrace{r-1, \ldots, r-1}_{d_{2}-d_{1}}, \ldots, \underbrace{1, \ldots, 1}_{d_{r}-d_{r-1}}) .
$$

That is the first $d_{1}$ rows has $r$-boxes, the next $d_{2}-d_{1}$ rows has $r-1$ boxes and continuing similarly we get only one box in the last $d_{r}-d_{r-1}$ rows.

Let $e_{1}, e_{2}, \ldots, e_{n}$ be the standard basis of $\mathbb{C}^{n}$, then

$$
v_{\chi}=\left(e_{1} \wedge \ldots \wedge e_{d_{1}}\right) \otimes\left(e_{1} \wedge \ldots \wedge e_{d_{2}}\right) \otimes \cdots \otimes\left(e_{1} \wedge \ldots \wedge e_{d_{r}}\right)
$$

in $\wedge^{d_{1}}\left(\mathbb{C}^{n}\right) \otimes \wedge^{d_{2}}\left(\mathbb{C}^{n}\right) \otimes \cdots \otimes \wedge^{d_{r}}\left(\mathbb{C}^{n}\right)$, is the highest weight vector of

$$
V_{\chi}=\left\langle G \cdot v_{\chi}\right\rangle
$$

by (cf. [FH13, Chapter 15]): We denote the affine cone over $\Sigma$ by

$$
\mathrm{a} \Sigma:=G \cdot \mathbb{C} v_{\chi} \subset V_{\chi}
$$

2.2. T-invariant Basis of $V_{\chi}$. To construct an explicit basis of $V_{\chi}$, we use the Deyrut's Construction of the Schur module [FH13] with highest weight $\chi$ which we describe below.

Let $\mathbb{C}[X]:=\mathbb{C}\left[x_{i j}\right]_{1 \leq i, j \leq n}$ be the polynomial ring in $n^{2}$ variables. Then $\mathbb{C}[X]$ is an $\mathrm{SL}(n, \mathbb{C})$-module via the following action

$$
A \cdot f(X)=f\left(A^{t} X\right) \text { for all } f \in \mathbb{C}[X] \text { and } A \in \mathrm{SL}(n, \mathbb{C}) .
$$

A semi-standard staircase tableau of length $r$ is a filling of the Young diagram of type (2) with entries from $\{1,2, \ldots, n\}$ which is weakly increasing across each row and strictly increasing along each column. We denote by $\mathcal{Y}$ the set of all semi standard staircase tableaux of the type (2).

Given a column vector $c=\left(c_{1}, c_{2}, \ldots, c_{l}\right)^{t}$ where $1 \leq c_{1}<c_{2}<\cdots<c_{l} \leq n$, let

$$
e_{c}:=\operatorname{det}\left(x_{i, c_{j}}\right)_{1 \leq i, j \leq l} .
$$

Let $Y \in \mathcal{Y}$. Let $c$ denote the entries of an arbitrary column in $Y$ and define

$$
e_{Y}:=\prod_{c} e_{c}
$$

Then $\left\langle e_{Y} \mid Y \in \mathcal{Y}\right\rangle$ is an irreducible sub-representation of $\mathbb{C}[X]$ with highest weight $\chi$ and highest weight vector $e_{Y_{0}}$ where $Y_{0}$ is the tableau in which the $i$-th row is filled with $i$ only (see in the following Young diagram). 


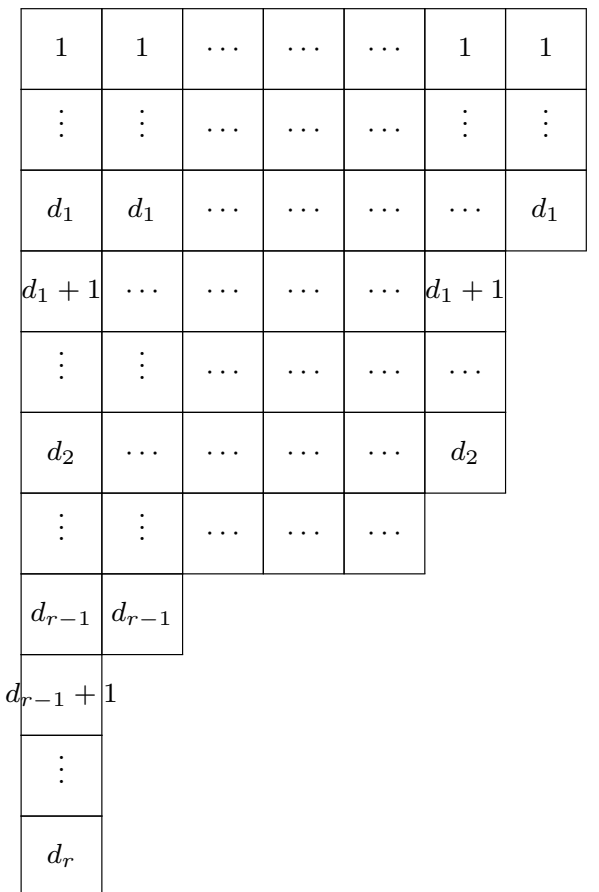

The Young tableau $Y_{0}$ corresponds to the highest weight

Thus, we define $V_{\chi}$ to be $\left\langle e_{Y} \mid Y \in \mathcal{Y}\right\rangle$. Each $e_{Y}$ is a weight vector under the action of $T$, with weight given by

$$
\prod_{i \in Y} t_{i}
$$

where the product runs over all the entries of $Y$. We thus have a $T$-invariant basis of $V_{\chi}$.

Example 2.1. There are 8 staircase tableaux of shape $(2,1)$ which are written below along with the corresponding weights.

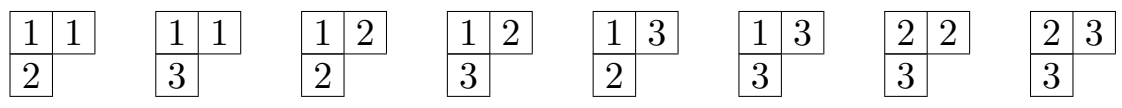

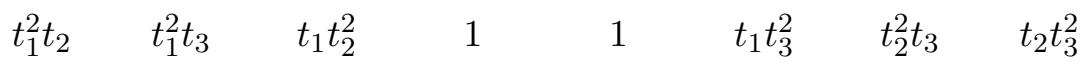

The weight vectors are all binomials in $x_{i j}$ 's. For instance, the highest weight vector is

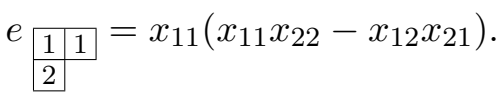

\section{Equivariant Cohomology of Weighted Flag orbifolds}

The aim of this section is to recall the definition of the weighted flag varieties, give their cell decomposition and to compute their cohomology.

It is well-known that the exponential map for a compact Lie group is always surjective. Thus a one-parameter subgroup in $G$ that is, $\mathbb{C}^{\times} \hookrightarrow G \times \mathbb{C}^{\times}$, can be found inside a maximal torus in $G$. Since all maximal tori are conjugate, so we consider the maximal torus to be $T$. 
Specifying weights on an affine cone over a given manifold is equivalent to choosing a one-parameter subgroup in $T$ (see [CR02 for a reference). Let $\rho \in \operatorname{Hom}\left(\mathbb{C}^{\times}, T\right)$ be one-parameter subgroup defined by

$$
\rho(t)=\operatorname{diag}\left(t^{w_{1}}, t^{w_{2}}, \ldots, t^{w_{n}}\right) \in G,
$$

where $w_{1}, \ldots, w_{n} \in \mathbb{Z}$. Take $u \in \mathbb{Z}_{\geq 0}$, we use $\rho$ and $u$ to make $V_{\chi}$ into a $G \times \mathbb{C}^{\times}$representation subject to the following action (of the second component of $G \times \mathbb{C}^{\times}$):

$$
t \cdot v=t^{u} \rho(t) v \forall v \in V_{\chi}, t \in \mathbb{C}^{\times} .
$$

We assume that this action has only positive weights, which can be done by taking $u$ to be sufficiently large CR02.

Definition 3.1. CR02] The weighted flag variety associated to the data $(\rho, u)$, is defined to be

$$
\mathrm{w} \Sigma:=\mathrm{a} \Sigma \backslash\{0\} / \mathbb{C}^{\times}=\mathrm{a} \Sigma^{\times} / \mathbb{C}^{\times} \subset \mathrm{w} \mathbb{P} V_{\chi}(\rho, u)
$$

where the quotient is taken by action of $\mathbb{C}^{\times}$on $V_{\chi}$, as defined above.

The weighted variety $\mathrm{w} \Sigma$ usually contains the quotient singularities due to the weights of their embeddings in $\mathrm{w} \mathbb{P} V_{\chi}(\rho, u)$ so we can also refer to them as weighted flag orbifolds. By definition, the obrifold $\mathrm{w} \Sigma$ has a natural residual action of

$$
T_{\mathrm{w}}:=\left(T \times \mathbb{C}^{\times}\right) / \mathbb{C}^{\times} \cong T
$$

such that the action of $T_{\mathrm{w}}$ extends to an action on the ambient space $\mathrm{w} \mathbb{P} V_{\chi}(\rho, u)$.

The $\mathbb{C}^{\times}$-action on $V_{\chi}$ via $\rho$ has each $e_{Y}$ as a weight vector with the weight $t^{w_{Y}}$ for $t \in \mathbb{C}^{\times}$, where

$$
w_{Y}=\sum_{i \in Y} w_{i}+u
$$

For the quotient of $\mathrm{a}^{\Sigma^{\times}}$by $\mathbb{C}^{\times}$to exist, we have to assume that $w_{Y}>0$ for all $Y \in \mathcal{Y}$, which, as remarked earlier, can be done by taking $u$ to be sufficiently large. The ambient weighted projective space of $\mathrm{w} \Sigma$ is thus

$$
\mathbb{P}\left(w_{Y} \mid Y \in \mathcal{Y}\right) \text {. }
$$

3.1. Open Charts and Cell Decomposition of $\mathrm{w} \Sigma$. We briefly recall the Bruhat order on the Weyl group $S_{n}$. Let $\sigma \in S_{n}$. We define the inversions of $\sigma$ to be

$$
\operatorname{Inv}(\sigma):=\{(i, j) \mid i<j, \sigma(i)>\sigma(j)\} .
$$

The length of $\sigma$ is defined by $l(\sigma):=|\operatorname{Inv}(\sigma)|$. Then, the permutation $\sigma_{0}=(n, n-$ $1, \ldots, 1)$ (written in the one-line notation) has the highest length $\left(\begin{array}{l}n \\ 2\end{array}\right)$. We say that $\sigma$ is covered by $\tau \in S_{n}$ and, denote by $\sigma \rightarrow \tau$, if there is a transposition $(i j) \operatorname{such}$ that $(i j) \cdot \sigma=\tau$ and $l(\tau)=l(\sigma)+1$. Note that $(i j)$ acts on $\sigma$ by switching indices $i$ and $j$ wherever they appear in $\sigma$. We say that $\sigma \prec \tau$, if there is a sequence $\sigma \rightarrow \sigma^{(0)} \rightarrow \sigma^{(1)} \rightarrow \cdots \rightarrow \sigma^{(s)} \rightarrow \tau$. The reflexive closure $\preceq$ of the relation induced by $\prec$ is a partial order on $S_{n}$ known as the Bruhat order. The permutation $\sigma_{0}$ is then the unique maximal element under this order, and the identity permutation id is the unique minimum. 
Let $W^{\prime}:=W / W_{P}$ be the quotient of the Weyl group $W$. The quotient $W^{\prime}$ corresponding to the highest weight (1) is given by

$$
W^{\prime}=S_{n} /\left(S_{d_{1}} \times S_{d_{2}-d_{1}} \times \cdots \times S_{d_{r}-d_{r-1}}\right) .
$$

Each coset $[\sigma] \in W^{\prime}$ has a unique representative of minimal length and the length of this representative is same in $W$ and $W^{\prime}$, i.e. $l([\sigma])=l(\sigma)$. We denote by $\operatorname{Inv}_{P}(\sigma)$ all inversions of $\sigma$ modulo $W_{P}$. Thus, the Bruhat order on $W$ induces the Bruhat order on $W^{\prime}$. The dimension of $\mathrm{w} \Sigma$ is equal to the length of longest element $\sigma_{0}^{\prime}$ in $W^{\prime}$. The set of minimal length representatives of all cosets in $W^{\prime}$ will be denoted by $W^{P}$.

Recall the Bruhat decomposition of $G$ is given by

$$
G=\coprod_{\sigma \in W} B \sigma B
$$

This induces the cell decomposition of $\Sigma$ into Schubert cells $\Omega_{\sigma}$ parameterized by $W^{P}$, i.e.,

$$
\Sigma=\coprod_{\sigma \in W^{P}} B \sigma P / P
$$

Let $e_{\sigma}$ denote the flag in $\Sigma$ corresponding to $\sigma \in W^{P}$ in the given basis $e_{1}, \ldots, e_{n}$. Explicitly,

$$
e_{\sigma}=\otimes_{i=1}^{r} \wedge_{j} e_{\sigma(j)},
$$

for $j \in I_{i}=\left\{\sigma(1), \ldots, \sigma\left(d_{i}\right)\right\}$. Each $\sigma \in W^{P}$ corresponds to a unique tableau $Y \in \mathcal{Y}$ as follows: fill the $i$ th column of $Y$ with entries from $\left\{\sigma(1), \ldots, \sigma\left(d_{r}-i+1\right)\right\}$ such that they are in increasing order. For instance, $\sigma=$ id corresponds to $Y_{0}$.

Example 3.2. Consider the complete flag variety $\mathcal{F} l(4)=G / B$. Then $\sigma=(1,4,2,3) \in S_{4}$ corresponds to

$$
e_{\sigma}=e_{1} \otimes e_{1} \wedge e_{4} \otimes e_{1} \wedge e_{4} \wedge e_{2}
$$

and the corresponding tableau $Y$ along with its weight is given as follows:

$$
\begin{aligned}
& \begin{array}{|l|l|l|}
\hline 1 & 1 & 1 \\
\hline
\end{array} \\
& \begin{array}{|l|l|}
\hline 2 & 4 \\
\hline 4 & \multicolumn{1}{|l}{} \\
\cline { 1 - 1 } &
\end{array} \\
& t_{1}^{3} t_{2} t_{4}^{2} \text {. }
\end{aligned}
$$

Consider the (generalized) $G$-equivariant Plücker embedding of flag variety

$$
\Sigma=G / P \hookrightarrow \mathbb{P}\left(V_{\chi}\right), \quad g \cdot e_{\mathrm{id}} \mapsto g \cdot e_{Y_{0}} .
$$

For each $\sigma \in W^{P}, e_{\sigma}$ corresponds to $e_{Y}$, thus we identify them. Let

$$
U_{\sigma}=\Sigma \cap U_{Y},
$$

where $U_{Y}$ is the open set of all points in $\mathbb{P}\left(V_{\chi}\right)$ with a non-zero $e_{Y^{-}}$coordinate. Then, by CR02 each $U_{\sigma}$ is $T$-equivariantly isomorphic to $\mathbb{C}^{l\left(\sigma_{0}^{\prime}\right)}$, where $\sigma_{0}^{\prime}$ is the maximal element 
in $W^{P}$. The union over all $\sigma \in W^{P}$ covers $\Sigma$. Moreover, the $T$-fixed points of $\Sigma$ are also parameterized by $W^{P}$ and their number is equal to $\left|W^{P}\right|$.

We now describe the open charts for $\mathrm{w} \Sigma$. Let

$$
K:=T \times \mathbb{C}^{\times}
$$

be the $n+1$-torus inside $G \times \mathbb{C}^{\times}$. Let

$$
\Pi_{\mathrm{w}}: \mathrm{a} \Sigma^{\times} \rightarrow \mathrm{w} \Sigma, \pi_{\mathrm{w}}: K \rightarrow T_{\mathrm{w}}
$$

be the quotient maps. For the straight flag manifold $\Sigma$, we denote the corresponding maps by $\Pi: \mathrm{a} \Sigma \rightarrow \Sigma$ and $\pi: K \rightarrow T$ respectively. By definition, $\Pi_{\mathrm{w}}$ makes a $\Sigma^{\times}$a $\pi_{\mathrm{w}}$-equivariant $\mathbb{C}^{\times}$-principal bundle over $\mathrm{w} \Sigma$. Indeed, a $\pi$-equivariant trivialization is given by

$$
\varphi_{\sigma}: \mathrm{a} U_{\sigma} \rightarrow U_{\sigma} \times \mathbb{C}^{\times}, x \mapsto\left(\Pi(x), x_{\sigma}\right),
$$

where a $U_{\sigma}:=\Pi^{-1}\left(U_{\sigma}\right)$ and the $K$-action on $U_{\sigma} \times \mathbb{C}^{\times}$is defined by factoring through $T$ via $\Pi$ on the first component, and by $\pi_{\sigma}$ (the projection on the $\sigma$-coordinate) on the second. Let

$$
\mathrm{w} U_{\sigma}:=\mathrm{a} U_{\sigma} / \mathbb{C}^{\times}
$$

and consider the map induced by $\varphi_{\sigma}$ between quotients

$$
\varphi_{\sigma}^{\mathrm{w}}: \mathrm{w} U_{\sigma} \rightarrow\left(U_{\sigma} \times \mathbb{C}^{\times}\right) / \mathbb{C}^{\times} .
$$

The stabilizer of the $\mathbb{C}^{\times}$on the second component of $U_{\sigma} \times \mathbb{C}^{\times}$is the cyclic group $\mu_{\sigma}:=$ $\left\{t \in \mathbb{C}^{\times} \mid t^{w_{\sigma}}=1\right\}$, where $w_{\sigma}=w_{Y}$ and thus, we obtain the following isomorphisms

$$
{ }_{\mathrm{w}} U_{\sigma} \cong U_{\sigma} / \mu_{\sigma} \cong \mathbb{C}^{l\left(\sigma_{0}^{\prime}\right)} / \mu_{\sigma},
$$

that are all $T_{\mathrm{w}}$-equivariant.

In a similar fashion, one can lift the Schubert cells $\Omega_{\sigma}$ via $\Pi$ to $a \Omega_{\sigma} \cong \Omega_{\sigma} \times \mathbb{C}^{\times} \subset \mathrm{a} \Sigma^{\times}$, which has same dimension as $\Omega_{\sigma}$ and its irreducibility follows from that of $\Omega_{\sigma}$. Then the $K$-invariant cell decomposition

$$
\mathrm{a} \Sigma^{\times}=\bigsqcup_{\sigma \in W^{P}} \mathrm{a} \Omega_{\sigma}
$$

is obtained from the Bruhat decomposition of $\Sigma=\bigsqcup_{\sigma \in W^{P}} \Omega_{\sigma}$. Similarly, the weighted flag variety $\mathrm{w} \Sigma$ has a $T_{\mathrm{w}}$-invariant weighted cell decomposition

$$
\mathrm{w} \Sigma=\bigsqcup_{\sigma \in W^{P}} \mathrm{w} \Omega_{\sigma} \text { such that } \mathrm{w} \Omega_{\sigma}:=\mathrm{a} \Omega_{\sigma} / \mathbb{C}^{\times} .
$$

Using the chart $\varphi_{\sigma}^{\mathrm{W}}$, we have

$$
\mathrm{w} \Omega_{\sigma} \cong \Omega_{\sigma} / \mu_{\sigma} \text {. }
$$

We will briefly recall the main idea of the Borel-Moore homology from [Ful97] and $\left[\mathrm{BM}^{+} 60\right]$. 
Definition 3.3. If $X$ is embedded in a closed subspace of Euclidean space $\mathbb{R}^{n}$, then its rational Borel-Moore homology is given by

$$
H_{i}^{B M}(X):=H^{n-i}\left(\mathbb{R}^{n}, \mathbb{R}^{n}-X\right) .
$$

In particular, for any oriented $n$-dimensional manifold $M$, we have

$$
H_{i}^{B M}(M)=H^{n-i}(M, M-M)=H^{n-i}(M),
$$

by [Ful97, Appendix B.2-(26)].

This formula can be extended to the case of any oriented, rationally smooth variety (see [CLS11, 11.4.3]). Given the cell decomposition of $\mathrm{w} \Sigma$, we obtain the following isomorphism [Ful97, Ex. 6, Appendix B.2]):

$$
H_{i}^{B M}(\mathrm{w} \Sigma) \cong \bigoplus_{\sigma \in W^{P}} H_{i}^{B M}\left(\mathrm{w} \Omega_{\sigma}\right)
$$

Now,

$$
\begin{aligned}
H_{i}^{B M}\left(\mathrm{w} \Omega_{\sigma}\right) & =H^{2\left(\operatorname{dim} \mathrm{w} \Omega_{\sigma}\right)-i}\left(\mathrm{w} \Omega_{\sigma}\right) \\
& =H^{2\left(\operatorname{dim} \mathrm{w} \Omega_{\sigma}\right)-i}\left(\Omega_{\sigma} / \mu_{\sigma}\right) \\
& \cong H^{2\left(\operatorname{dim} \mathrm{w} \Omega_{\sigma}\right)-i}\left(\Omega_{\sigma}\right)^{\mu_{\sigma}} \\
& \cong H^{2\left(\operatorname{dim} \mathrm{w} \Omega_{\sigma}\right)-i}\left(\Omega_{\sigma}\right)
\end{aligned}
$$

where the first equality follows from the statements given above, since each cell is locally the quotient of a Euclidean space modulo a finite group, hence rationally smooth [CLS11, 11.4.4]. The first isomorphism follows from the classical result (see [Bre72, Theorem 2.4, Chapter III]). The second isomorphism comes from the fact that as $\mu_{\sigma}$ acts through the connected group $\mathbb{C}^{\times}$, hence its induced action on cohomology must be trivial. By Equations (4) and (5), the odd rational cohomology of $\mathrm{w} \Sigma$ vanishes.

\section{Proposition 3.4.}

$$
H^{i}(\mathrm{w} \Sigma)=\left\{\begin{array}{cc}
\mathbb{Q}^{h} & i=2 k \\
0 & \text { otherwise }
\end{array}\right.
$$

where $h$ is the number of elements in $W^{P}$ of length $k$.

Proof. The proof follows from (4) and (5).

Thus we have the following theorem:

Theorem 3.5. There is an $H^{*}\left(B T_{\mathrm{w}}\right)$-module isomorphism

$$
H_{T_{\mathrm{w}}}^{*}(\mathrm{w} \Sigma) \cong H^{*}\left(B T_{\mathrm{w}}\right) \otimes_{\mathbb{Q}} H^{*}(\mathrm{w} \Sigma),
$$

where $H_{T_{\mathrm{w}}}^{*}($.$) is the T_{\mathrm{w}}$-equivariant cohomology and $B T_{\mathrm{w}}$ is the classifying space of $T_{\mathrm{w}}$ (see $\left.\left[\mathrm{BBF}^{+} 60\right]\right)$. In fact, $H_{T_{\mathrm{w}}}^{*}(\mathrm{w} \Sigma)$ is a free module.

Proof. The torus $T_{\mathrm{w}}$ is connected and the odd cohomology of $\mathrm{w} \Sigma$ vanishes. The result follows from [GKM97, Theorem 14.1]. 
Example 3.6. Consider the flag variety

$$
\Sigma=G L(4, \mathbb{C}) / P=\left\{0 \subset V_{d_{1}} \subset V_{d_{2}} \subset \mathbb{C}^{4}\right\}
$$

where $d_{1}=1$ and $d_{2}=3$. Here the Weyl group $S_{4}$ has the corresponding Parabolic subgroup $W_{P}=\left\langle s_{2}\right\rangle$. The set of minimal length representatives of cosets in $W^{P}$ is

$$
W^{P}=\left\{\mathrm{id}, s_{1}, s_{3}, s_{1} s_{3}, s_{2} s_{1}, s_{2} s_{3}, s_{2} s_{1} s_{3}, s_{1} s_{2} s_{3}, s_{3} s_{2} s_{1}, s_{3} s_{2} s_{3} s_{1}, s_{1} s_{2} s_{3} s_{1}, s_{3} s_{1} s_{2} s_{3} s_{1}\right\} .
$$

The variety $\Sigma$ is five dimensional, thus by using Proposition 3.4 , we have

$$
H^{0}(\mathrm{w} \Sigma)=H^{10}(\mathrm{w} \Sigma)=\mathbb{Q}, H^{2}(\mathrm{w} \Sigma)=H^{8}(\mathrm{w} \Sigma)=\mathbb{Q}^{2} \text { and } H^{4}(\mathrm{w} \Sigma)=H^{6}(\mathrm{w} \Sigma)=\mathbb{Q}^{3} .
$$

3.2. Equivariant Cohomology Ring of $\mathrm{w} \Sigma$. Since $a \Sigma^{\times}$is a smooth quasi-projective variety inside $\mathbb{C}^{|\mathcal{Y}|}$, it has a natural structure of a symplectic manifold given by $\omega=$ $\sum_{Y \in \mathcal{Y}} d x_{Y} \wedge d \bar{x}_{Y}$. Following [Kir84, Section 9], we describe $\mathrm{w} \Sigma$ as a quotient of a compact real symplectic submanifold of $\mathrm{a}^{\times}$by the hamiltonian action of compact real torus inside $\mathbb{C}^{\times}$.

Let $S^{1}=\left\{z \in \mathbb{C}^{\times}|| z \mid=1\right\}, S_{T}:=\left(S^{1}\right)^{n}, S_{K}:=\left(S^{1}\right)^{n+1}$ be the real tori inside $\mathbb{C}^{\times}, T$ and $K$ respectively. We define the real torus inside $T_{\mathrm{w}}$ to be $S_{T_{\mathrm{w}}}:=S_{K} / S^{1}$.

Lemma 3.7. There is compact symplectic submanifold $M$ of $a \Sigma^{\times}$such that $\mathrm{a} \Sigma^{\times} K$ equivariantly deformation retracts to $M$ and

$$
M / S^{1} \cong \mathrm{w} \Sigma .
$$

Proof. The $S^{1}$ action on $V_{\chi} \cong \mathbb{R}^{2|\mathcal{Y}|}$ factors through the $\mathbb{C}^{\times}$action defined earlier, and since a $\Sigma^{\times}$is $S^{1}$-invariant, the action restricts to $a \Sigma^{\times}$. The associated vector field for this action is

$$
X=\sum_{Y \in \mathcal{Y}} w_{Y}\left(-x_{Y}^{2} \partial x_{Y}^{1}+x_{Y}^{1} \partial x_{Y}^{2}\right)
$$

where $x_{Y}^{1}=\Re\left(x_{Y}\right), x_{Y}^{2}=\Im\left(x_{Y}\right)$ and $w_{Y}$ is as defined in (3).

We identify $\mathbb{R}$ with both the Lie algebra of $S^{1}$ and its dual. The duality pairing then becomes the standard inner product on $\mathbb{R}$. Let $\mu: V_{\chi} \rightarrow \mathbb{C}$ be defined by

$$
\mu(x)=i \sum_{Y \in \mathcal{Y}} w_{Y}\left|x_{Y}\right|^{2} .
$$

Below we check that $\mu$ is a moment map with respect to which $X$ is Hamiltonian.

$$
\begin{aligned}
\iota_{X}(\omega) & =2 i \sum_{Y \in \mathcal{Y}} w_{Y}\left(x_{Y}^{2} d x_{Y}^{2}+x_{Y}^{1} d x_{Y}^{1}\right) \\
& =2 i \sum_{Y \in \mathcal{Y}} \frac{1}{2} w_{Y} d\left(\left(x_{Y}^{2}\right)^{2}+\left(x_{Y}^{1}\right)^{2}\right) \\
& =i \sum_{Y \in \mathcal{Y}} d\left(w_{Y}\left|x_{Y}\right|^{2}\right) \\
& =d \mu .
\end{aligned}
$$

Since a $\Sigma^{\times}$is an $S^{1}$-invariant symplectic submanifold in $V_{\chi}$, the restriction of $\mu$ on a $\Sigma^{\times}$ gives us a moment map with respect to which the induced action on $a \Sigma^{\times}$is Hamiltonian. 
Let $M=\mu^{-1}(\lambda)$ be a level surface for any regular value $\lambda$ of $\mu$. Then, $M$ is a smooth $S_{K^{-}}$invariant submanifold inside a $\Sigma^{\times}$. The map

$$
f: a \Sigma^{\times} \times[0,1] \rightarrow \mathrm{a}^{\times}
$$

given by

$$
\left(\left(x_{Y}\right)_{Y \in \mathcal{Y}}, t\right) \mapsto\left((1+t(\sqrt{\lambda / \mu(x)}-1))_{Y}\right)_{Y \in \mathcal{Y}}
$$

is easily checked to be a deformation retraction from $\mathrm{a}^{\Sigma^{\times}}$to $M$. Furthermore, this deformation retraction is equivariant with respect to the $S_{K}$ action that is, each $f_{t}$ is a

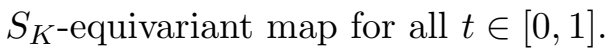

Since a $\Sigma^{\times}$is $S_{K}$-invariant, the quotient ${ }_{\mathrm{w}} \Sigma^{\times}$is $S_{T_{\mathrm{w}}}$-invariant. As the inclusion $\iota: M \rightarrow$ a $\Sigma^{\times}$is equivariant with respect to the inclusion $\left(S^{1}\right)^{n+1} \hookrightarrow K$, the induced quotient map $\bar{\iota}: M / S^{1} \rightarrow \mathrm{w} \Sigma$ will be equivariant with respect to the inclusion $S_{T_{\mathrm{w}}} \hookrightarrow T_{\mathrm{w}}$. Furthermore, it is a homeomorphism as its inverse is given by

$$
\left(x_{Y}\right)_{Y \in \mathcal{Y}} \rightarrow\left(x_{Y} \sqrt{\lambda / \mu(x)}\right)_{Y \in \mathcal{Y}},
$$

which can easily be seen to be continuous.

Using the above Lemma we obtain the following isomorphism which gives us the equivariant cohomology ring of $\mathrm{w} \Sigma$.

Theorem 3.8. The map $\bar{\iota}^{*}: H_{T_{\mathrm{w}}}^{*}(\mathrm{w} \Sigma) \rightarrow H_{S_{T_{\mathrm{w}}}}^{*}\left(M / S^{1}\right)$ is a ring isomorphism.

\section{SCHUbert CLASSES AND GKM DESCRIPTIONS}

4.1. Weighted Schubert classes. Let us denote by $\tilde{\xi}_{\sigma}$ the equivariant Schubert classes forming distinguished $H_{T}^{*}(B T)$-module basis for $H_{T}^{*}(\Sigma)$, see $\left[\mathrm{BBF}^{+} 60\right]$. The classes $\tilde{\xi}_{\sigma}$ are obtained as the equivariant fundamental classes of the $T$-invariant Schubert varieties $X_{\sigma}$ which are closures of the corresponding Schubert cells $\Omega_{\sigma}$. We start with the following diagram.

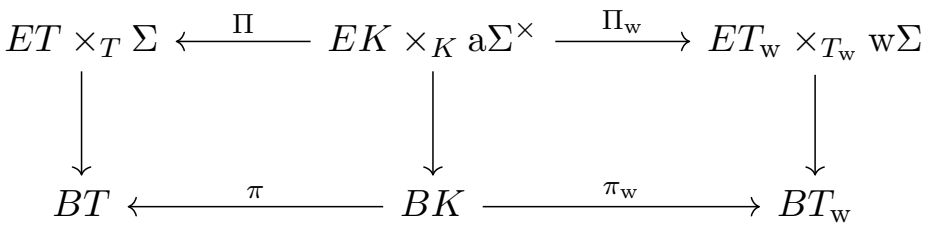

Applying the cohomology functor, we get the following diagram:

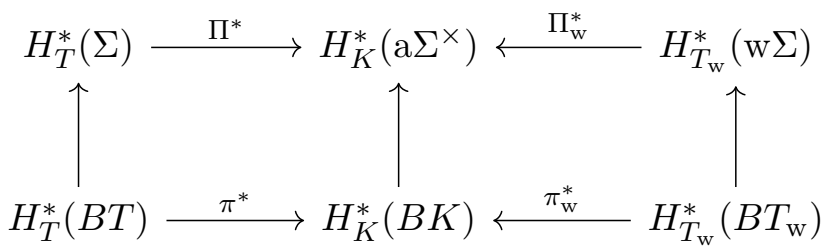

We take $\tilde{\xi}_{\sigma}=\left[\Omega_{\sigma}\right]_{T} \in H_{T}^{2 l(\sigma)}(\Sigma)$, then the Schubert cycles for the punctured affine cone $\mathrm{a} \Sigma^{\times}$and the weighted flag orbifold $\mathrm{w} \Sigma$, can be described via maps $\Pi^{*}$ and $\Pi_{\mathrm{w}}^{*}$ respectively as follows, by the same argument as in [AM15a] for the case of Grassmannians:

$$
\mathrm{a} \tilde{\xi}_{\sigma}:=\Pi^{*}\left(\tilde{\xi}_{\sigma}\right) \in H_{K}^{2 l(\sigma)}\left(\mathrm{a} \Sigma^{\times}\right) \quad \mathrm{w} \tilde{\xi}_{\sigma}:=\left(\Pi_{\mathrm{w}}^{*}\right)^{-1}\left(\mathrm{a} \tilde{\xi}_{\sigma}\right) \in H_{T_{\mathrm{w}}}^{2 l(\sigma)}(\mathrm{w} \Sigma)
$$


4.2. GKM description of $H_{T_{\mathrm{w}}}^{*}(\mathrm{w} \Sigma)$. Let the $T$-equivariant cohomology $H^{*}(B T)$ of a point be denoted by $\mathbb{Q}\left[T^{*}\right] \cong \mathbb{Q}\left[y_{1}, \ldots, y_{n}\right]$. Let

$$
\mathbb{Q}\left[K^{*}\right]:=H^{*}(B K)=\mathbb{Q}\left[y_{1}, \ldots, y_{n}, z\right] .
$$

Since $K=T \times \mathbb{C}^{\times}$, one can take the basis of $\mathbb{Z}$-linear functionals $\left\{y_{1}, \ldots, y_{n}, z\right\}$ on the integral sublattice of $\operatorname{Lie}(K)^{*}$.

Define $\rho^{*}: \operatorname{Lie}(K)^{*} \rightarrow \operatorname{Lie}\left(\mathbb{C}^{\times}\right)^{*}=\mathbb{Q}[\delta]$ by

$$
y_{i} \mapsto w_{i} \delta \text { and } z \mapsto-u \delta
$$

By definition of $T_{\mathrm{w}}$ we have $\operatorname{ker}\left(\rho^{*}\right)=\operatorname{Lie}\left(T_{\mathrm{w}}\right)^{*}$. Let $y_{1}^{\mathrm{w}}, \ldots, y_{n}^{\mathrm{w}}$ denote the $\mathbb{Z}$-basis of $\operatorname{Lie}\left(T_{\mathrm{w}}\right)^{*}$. Since $T_{\mathrm{w}}$ is defined as the quotient of $K$, thus we define $y_{i}^{\mathrm{w}}$ as elements of $\operatorname{ker}\left(\rho^{*}\right)$ by

$$
y_{i}^{\mathrm{w}}:=y_{i}+\frac{w_{i}}{u} z, \text { for all } i=1, \ldots, n
$$

Let

$$
\mathbb{Q}\left[T_{\mathrm{w}}^{*}\right]:=H^{*}\left(B T_{\mathrm{w}}\right)=\mathbb{Q}\left[y_{1}^{\mathrm{w}}, \ldots, y_{n}^{\mathrm{w}}\right] \subset \mathbb{Q}\left[K^{*}\right] .
$$

For each $\sigma \in W^{P}$, we use the following notation in the rest of the paper,

$$
\begin{aligned}
& y_{\sigma}:=r\left(y_{\sigma_{1}}+\cdots+y_{\sigma_{d_{1}}}\right)+(r-1)\left(y_{\sigma_{d_{1}+1}}+\cdots+y_{\sigma_{d_{2}}}\right)+\cdots+\left(y_{\sigma_{d_{r-1}+1}}+\cdots+y_{\sigma_{d_{r}}}\right), \\
& y_{\sigma}^{\mathrm{w}}:=r\left(y_{\sigma_{1}}^{\mathrm{w}}+\cdots+y_{\sigma_{d_{1}}}^{\mathrm{w}}\right)+(r-1)\left(y_{\sigma_{d_{1}+1}}^{\mathrm{w}}+\cdots+y_{\sigma_{d_{2}}}^{\mathrm{w}}\right)+\cdots+\left(y_{\sigma_{d_{r-1}+1}}^{\mathrm{w}}+\cdots+y_{\sigma_{d_{r}}}^{\mathrm{w}}\right), \\
& w_{\sigma}:=r\left(w_{\sigma_{1}}+\cdots+w_{\sigma_{d_{1}}}\right)+(r-1)\left(w_{\sigma_{d_{1}+1}}+\cdots+w_{\sigma_{d_{2}}}\right)+\cdots+\left(w_{\sigma_{d_{r-1}+1}}+\cdots+w_{\sigma_{d_{r}}}\right)+u,
\end{aligned}
$$

where $\sigma_{i}=\sigma(i)$. The fixed points under the action of $T$ on $\Sigma$ are $\left[e_{\sigma}\right]$, for $\sigma \in W^{P}$. We also denote the $T_{\mathrm{w}}$-fixed points of $\mathrm{w} \Sigma$ by the same notation $\left[e_{\sigma}\right]$. By equivariant formality of $\Sigma$ (see for instance in [GHZ06]), the restriction map to the fixed points is injective,

$$
H_{T}^{*}(\Sigma) \rightarrow \bigoplus_{\sigma \in W^{P}} \mathbb{Q}\left[T^{*}\right] ; \quad \alpha \mapsto\left(\alpha_{\sigma}\right)_{\sigma \in W^{P}}
$$

By [GKM97, the image of the above map is given by

$$
\left\{\alpha=\left(\alpha_{\sigma}\right)_{\sigma \in W^{P}} \in \bigoplus_{\sigma \in W^{P}} \mathbb{Q}\left[T^{*}\right] \mid \alpha_{\sigma}-\alpha_{\tau} \in\left\langle y_{i}-y_{j}\right\rangle=\left\langle y_{\sigma}-y_{\tau}\right\rangle \text {; whenever } \sigma=(i j) \tau\right\} \text {. }
$$

Since the fixed points of the $T_{\mathrm{w}}$-action are the images of $\left[e_{\sigma}\right]$ in $\mathrm{w} \Sigma$ and given the isomorphism $H_{T_{\mathrm{w}}}^{*}\left(\left[e_{\sigma}\right]\right) \cong \mathbb{Q}\left[T_{\mathrm{w}}^{*}\right]$, we have the following restriction map

$$
H_{T_{\mathrm{w}}}^{*}(\mathrm{w} \Sigma) \rightarrow \bigoplus_{\sigma \in W^{P}} \mathbb{Q}\left[T_{\mathrm{w}}^{*}\right] ; \quad \gamma \mapsto\left(\gamma_{\sigma}\right)_{\sigma \in W^{P}}
$$

For any $\sigma \in W^{P}$, the image of $\left[e_{\sigma}\right]$ in a $\Sigma^{\times}$is a $\mathbb{C}^{\times}$-orbit of $e_{\sigma}$. Denote by $K_{\sigma}$, kernel of the map $K \rightarrow \mathbb{C}^{\times}$, given by

$$
\left(t_{1}, \ldots, t_{n}, s\right) \mapsto s^{-1} t^{\sigma} ; t^{\sigma}=\left(t_{\sigma_{1}} \ldots t_{\sigma_{d_{1}}}\right)^{r}\left(t_{\sigma_{d_{1}+1}} \ldots t_{\sigma_{d_{2}}}\right)^{r-1} \ldots\left(t_{\sigma_{d_{r-1}+1}} \ldots t_{\sigma_{d_{r}}}\right) .
$$


By definition, $K_{\sigma}$ is the set of those elements which fix $e_{\sigma} \in \mathrm{w} \Sigma$. Thus the $K_{\sigma}$-equivariant cohomology of the fixed point $\left[e_{\sigma}\right]$ is

$$
H_{K_{\sigma}}^{*}\left(e_{\sigma}\right) \cong \mathbb{Q}\left[K_{\sigma}^{*}\right],
$$

where $\mathbb{Q}\left[K_{\sigma}^{*}\right]=\mathbb{Q}[K] /\left\langle y_{\sigma}-z\right\rangle$. Indeed, this is true because $K_{\sigma}$ is the isotropy subgroup of $\sigma$ in $K$ and due to the following isomorphism

$$
\operatorname{Lie}\left(K_{\sigma}\right)^{*} \cong \operatorname{Lie}(K)^{*} /\left\langle y_{\sigma}-z\right\rangle .
$$

The restriction map for $a \Sigma^{\times}$thus reads

$$
H_{K}^{*}\left(\mathrm{a} \Sigma^{\times}\right) \rightarrow \bigoplus_{\sigma \in W^{P}} \mathbb{Q}\left[K_{\sigma}^{\star}\right] \cong \bigoplus_{\sigma \in W^{P}} \mathbb{Q}\left[K^{*}\right] /\left\langle y_{\sigma}-z\right\rangle ; \quad Q \mapsto\left(Q_{\sigma}\right)_{\sigma \in W^{P}}
$$

The map (10) is injective due to injectivity of (8) and (9) along with the commutativity of the following diagram

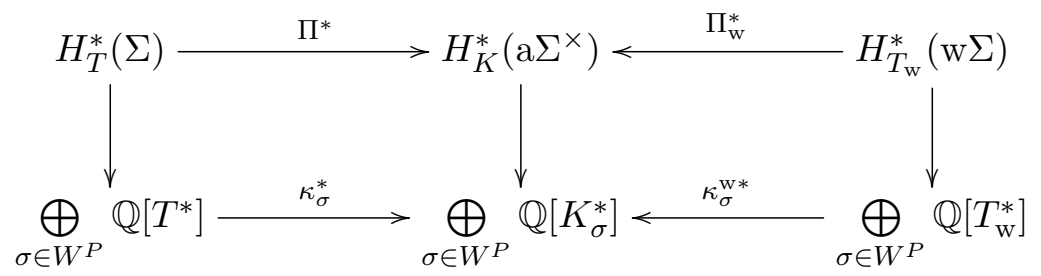

where the maps giving the bottom arrows are induced by $\kappa_{\sigma}: K_{\sigma} \hookrightarrow K \rightarrow T$ and $\kappa_{\sigma}^{\mathrm{w}}: K_{\sigma} \hookrightarrow K \rightarrow T_{\mathrm{w}}$ defined by sending each element to its class modulo $\left\langle y_{\sigma}-z\right\rangle$.

Theorem 4.1. The equivariant cohomology of $\mathrm{w} \Sigma$ has the following GKM description

$$
\left\{\gamma=\left(\gamma_{\sigma}\right)_{\sigma \in W^{P}} \in \bigoplus_{\sigma \in W^{P}} \mathbb{Q}\left[T_{\mathrm{w}}^{*}\right] \mid \gamma_{\sigma}-\gamma_{\tau} \in\left\langle w_{\tau} y_{\sigma}^{\mathrm{w}}-w_{\sigma} y_{\tau}^{\mathrm{w}}\right\rangle ; \text { whenever } \sigma=(i j) \tau\right\} .
$$

Proof. In order to show the equivalence of GKM conditions we need to check if the following isomorphism is valid:

$$
\mathbb{Q}\left[T_{\mathrm{w}}^{*}\right] /\left\langle w_{\tau} y_{\sigma}^{\mathrm{w}}-w_{\sigma} y_{\tau}^{\mathrm{w}}\right\rangle \cong \mathbb{Q}\left[K^{*}\right] /\left\langle y_{\sigma}-z, y_{\tau}-z\right\rangle .
$$

The first of the following isomorphisms is implied by the isomorphism $\kappa_{\sigma}^{\mathrm{w}^{*}}$,

$$
\mathbb{Q}\left[T_{\mathrm{w}}^{*}\right] /\left\langle w_{\tau} y_{\sigma}^{\mathrm{w}}-w_{\sigma} y_{\tau}^{\mathrm{w}}\right\rangle \cong \mathbb{Q}\left[K^{*}\right] /\left\langle y_{\sigma}-z, w_{\tau} y_{\sigma}^{\mathrm{w}}-w_{\sigma} y_{\tau}^{\mathrm{w}}\right\rangle \cong \mathbb{Q}\left[K^{*}\right] /\left\langle y_{\sigma}-z, y_{\tau}-z\right\rangle .
$$

The second isomorphism follows because

$$
w_{\tau} y_{\sigma}^{\mathrm{w}}-w_{\sigma} y_{\tau}^{\mathrm{w}}=-w_{\sigma}\left(y_{\tau}-z\right) \text { in } \mathbb{Q}\left[K^{*}\right] /\left\langle y_{\sigma}-z\right\rangle .
$$

Theorem 4.2. The equivariant cohomology of $\mathrm{a}^{\times}$has the following GKM description $\left\{Q=\left(Q_{\sigma}\right)_{\sigma \in W^{P}} \in \bigoplus_{\sigma \in W^{P}} \mathbb{Q}\left[K^{*}\right] \mid Q_{\sigma}=Q_{\tau} \quad\right.$ in $\mathbb{Q}\left[K^{*}\right] /\left\langle y_{\sigma}-z, y_{\tau}-z\right\rangle ;$ whenever $\left.\sigma=(i j) \tau\right\}$. 
We have proven the equivalence of GKM conditions for $\kappa_{\sigma}^{\mathrm{w} *}$ above, the equivalence for $\kappa_{\sigma}^{*}$ follows as a special case of Theorem 4.1.

Next we express the Schubert classes using the GKM descriptions stated above. It is known for partial flag manifold $\Sigma$ Tym09, Corollary 4.5], that for any $\sigma \in W^{P}$,

$$
\left.\tilde{\xi}_{\sigma}\right|_{\tau}=\left\{\begin{array}{cl}
\prod_{(i, j) \in \operatorname{Inv}_{P}(\sigma)}\left(y_{j}-y_{i}\right) & \tau=\sigma \\
0 & \tau \nsucceq \sigma
\end{array}\right.
$$

Using the definitions in (7), we write the following equivalent description

$$
\left.\tilde{\xi}_{\sigma}\right|_{\tau}=\left\{\begin{array}{cl}
h_{\sigma} \prod_{(i, j) \in \operatorname{Inv}_{P}(\sigma)}\left(y_{\sigma}-y_{(i j) \sigma}\right) & \tau=\sigma \\
0 & \tau \nsucceq \sigma
\end{array},\right.
$$

where $h_{\sigma}=\prod_{(i, j) \in \operatorname{Inv}_{P}(\sigma)}(q-p)^{-1}$, where $p$ and $q$ are chosen so that $i \in\left[d_{p}+1, d_{p+1}\right]$ and $j \in\left[d_{q}+1, d_{q+1}\right]$.

This description along with the commutative diagram (11) and the formulae for Schubert classes in $\mathrm{a}^{\times}$and $\mathrm{w} \Sigma$ imply the following two propositions.

Proposition 4.3. The Schubert classes in $\mathrm{a} \Sigma^{\times}$have the following GKM description

$$
\left.\mathrm{a} \tilde{\xi}_{\sigma}\right|_{\tau}=\left\{\begin{array}{cl}
h_{\sigma} \prod_{(i, j) \in \operatorname{Inv}_{P}(\sigma)}\left(z-y_{(i j) \sigma}\right) & \tau=\sigma \\
0 & \tau \nsucceq \sigma
\end{array} .\right.
$$

Proposition 4.4. The Schubert classes in $\mathrm{w} \Sigma$ have the following GKM description

$$
\left.\mathrm{w}_{\tilde{\xi}_{\sigma}}\right|_{\tau}=\left\{\begin{array}{cc}
h_{\sigma} \prod_{(i, j) \in \operatorname{Inv}_{P}(\sigma)}\left(\frac{w_{(i j) \sigma}}{w_{\sigma}} y_{\sigma}^{\mathrm{w}}-y_{(i j) \sigma}^{\mathrm{w}}\right) & \tau=\sigma \\
0 & \tau \nsucceq \sigma
\end{array} .\right.
$$

For simple transpositions $s_{d} \in W^{P}$ one has the simple formula,

$$
\left.\tilde{\xi}_{s_{d}}\right|_{\tau}=\sum_{j=1}^{d} y_{\tau(j)}-\sum_{j=1}^{d} y_{j}
$$

This can easily be checked for all $\tau \in W^{P}$. Using the upper triangularity of weighted Schubert classes, as above, one obtains a basis for the equivariant cohomology of the weighted flag orbifold $\mathrm{w} \Sigma$.

Proposition 4.5. A $H^{*}\left(B T_{\mathrm{w}}\right)$-module basis of $H_{T_{\mathrm{w}}}^{*}(\mathrm{w} \Sigma)$ is given by the weighted Schubert classes $\left\{\mathrm{w} \tilde{\xi}_{\sigma}\right\}_{\sigma \in W^{P}}$.

\section{Chevalley's formula FOR $\mathrm{w} \Sigma$}

Chevalley's formula computes the product of any Schubert cycle with a divisor class corresponding to a simple root. We compute the weighted version of the Chevalley's formula. We also give the polynomial description for the equivariant cohomology ring of $\mathrm{w} \Sigma$ and polynomial representative for a weighted Schubert class, inspired by [AM15b]. 
5.1. Weighted Chevalley's Formula. Using (6) and the Schubert class (16) for simple transposition $s_{d} \in W^{P}$, we have

$$
\left.\mathrm{a} \tilde{\xi}_{s_{d}}\right|_{\tau}=\sum_{j=1}^{d}\left(y_{\tau(j)}^{\mathrm{w}}-y_{j}^{\mathrm{w}}-\frac{w_{\tau(j)}-w_{j}}{u} z\right) .
$$

We give an equivariant Chevalley's formula for the weighted flag orbifold as follows.

Theorem 5.1. For any simple reflection $s_{d} \in W^{P}$ and $\sigma \in W^{P}$ the weighted Chevalley's formula is given by

$$
\mathrm{w} \tilde{\xi}_{s_{d}} \mathrm{w} \tilde{\xi}_{\sigma}=\sum_{j=1}^{d}\left(y_{\sigma(j)}^{\mathrm{w}}-y_{j}^{\mathrm{w}}-\frac{w_{\sigma(j)}-w_{j}}{u} z\right) \mathrm{w} \tilde{\xi}_{\sigma}+\sum_{\substack{i \leq d<j \\ l((i j) \sigma)=l(\sigma)+1}} \mathrm{w} \tilde{\xi}_{(i j) \sigma} .
$$

Proof. We have the following formula of Kostant-Kumar [KK86, Proposition 4.30] in the case of partial flag variety $\Sigma$

$$
\tilde{\xi}_{s_{d}} \tilde{\xi}_{\sigma}=\tilde{\xi}_{s_{d}} \mid \sigma \tilde{\xi}_{\sigma}+\sum_{\substack{i \leq d_{k}<j \\ l((i j) \sigma)=l(\sigma)+1}} \tilde{\xi}_{(i j) \sigma} .
$$

By applying $\Pi^{*}$ to the above equation we get

$$
\mathrm{a} \tilde{\xi}_{s_{d}} \mathrm{a} \tilde{\xi}_{\sigma}=\mathrm{a} \tilde{\xi}_{s_{d}} \mid \sigma \mathrm{a} \tilde{\xi}_{\sigma}+\sum_{\substack{i \leq d_{k}<j \\ l((i j) \sigma)=l(\sigma)+1}} \mathrm{a} \tilde{\xi}_{(i j) \sigma} .
$$

An application of $\left(\Pi_{\mathrm{w}}^{*}\right)^{-1}$ along with the use of 17$)$ we obtain the result.

We define a special Schubert class $\tilde{\xi}_{\text {div }}$ given by $(19)$, which corresponds to length one elements $s_{d}$ in $W^{P}$. We use it to prove Proposition 5.5.

$$
\tilde{\xi}_{\text {div }}:=\sum_{i=1}^{r} \tilde{\xi}_{s_{i}} .
$$

Recall that for $\sigma \in W^{P}$,

$$
y_{\sigma}=r\left(y_{\sigma_{1}}+\cdots+y_{\sigma_{d_{1}}}\right)+(r-1)\left(y_{\sigma_{d_{1}+1}}+\cdots+y_{\sigma_{d_{2}}}\right)+\cdots+\left(y_{\sigma_{d_{r-1}+1}}+\cdots+y_{\sigma_{d_{r}}}\right)
$$

By using the GKM description for simple transpositions and (17), we get

$$
\begin{aligned}
\left.\tilde{\xi}_{\text {div }}\right|_{\tau} & =\left.\tilde{\xi}_{s_{d_{1}}}\right|_{\tau}+\cdots+\left.\tilde{\xi}_{s_{d_{r}}}\right|_{\tau} \\
& =\sum_{j=1}^{d_{1}} y_{\tau(j)}-\sum_{j=1}^{d_{1}} y_{j}+\cdots+\sum_{j=1}^{d_{r}} y_{\tau(j)}-\sum_{j=1}^{d_{r}} y_{j} \\
& =y_{\tau}-y_{\mathrm{id}}
\end{aligned}
$$

Now using

$$
y_{\tau}-y_{\text {id }}=z-y_{\text {id }} \text { in } \mathbb{Q}\left[K^{*}\right] /\left\langle y_{\tau}-z\right\rangle,
$$

we have

$$
\left.\mathrm{a} \tilde{\xi}_{\operatorname{div}}\right|_{\tau}=z-y_{\text {id }}
$$


5.2. Weighted Schubert Polynomials. To define the weighted Schubert polynomials, we recall the definition of double (equivariant) Schubert polynomial. To give the description, we recall the following definition and notations which can also be found in [Man01].

Definition 5.2. Let $x_{i}, i=1, \ldots, n$ and $b_{j}, j \in \mathbb{N}$ be indeterminates. Let $\mathbb{Q}[x]:=$ $\mathbb{Q}\left[x_{1}, \ldots, x_{n}\right]$ be the polynomial ring over $\mathbb{Q}$. Let $\mathbb{Q}[x, b]:=\mathbb{Q}[x] \otimes_{\mathbb{Q}} \mathbb{Q}[b]$. For each $\sigma \in S_{\infty}:=\cup_{k \geq 1} S_{k}$, the double (equivariant) Schubert polynomials $\mathfrak{S}_{\sigma}$ are defined as follows:

$$
\mathfrak{S}_{\sigma}(x, b):=\sum_{\substack{\sigma=\tau^{-1} \mu \\ l(\sigma)=l(\tau)+l(\mu)}} \mathfrak{S}_{\tau}(x) \mathfrak{S}_{\mu}(-b)
$$

where $\mathfrak{S}_{\tau}$ is the usual Schubert polynomial.

The usual and double Schubert polynomials have the following well-known properties:

(1) $\mathfrak{S}_{\sigma_{0}}(x)=x_{1}^{n-1} x_{2}^{n-2} \ldots x_{n-1}$ and $\mathfrak{S}_{s_{d}}(x)=x_{1}+x_{2}+\cdots+x_{d}$.

(2) $\mathfrak{S}_{\sigma_{0}}(x, b)=\prod_{i+j \leq n}\left(x_{i}-b_{j}\right)$, where $\sigma_{0}$ is the unique maximal element in $S_{n}$.

(3) $\mathfrak{S}_{\mathrm{id}}(x)=\mathfrak{S}_{\mathrm{id}}(x, b)=1$.

It is well known that the set $\left\{\mathfrak{S}_{\sigma}\right\}_{\sigma \in S_{\infty}}$ forms a $\mathbb{Q}[b]$-basis of $\mathbb{Q}[x, b]$. We can regard $H_{T}^{*} \Sigma$ as a $\mathbb{Q}[b]$-module by using the projection map $\mathbb{Q}[b] \rightarrow H^{*}(B T)=\mathbb{Q}\left[y_{1}, \ldots, y_{n}\right]$ that sends $b_{j}$ to $y_{j}$, for $j=1, \ldots, n$ and to 0 for $j>n$. Thus we have a surjective homomorphism

$$
\theta: \mathbb{Q}[x, b] \rightarrow H_{T}^{*}(\Sigma)
$$

of algebras over $\mathbb{Q}[b]$ which maps the polynomial $\mathfrak{S}_{\sigma}$ to the Schubert class $\tilde{\xi}_{\sigma^{-1}}$ of codimension $l(\sigma)$, if $\sigma \in W^{P}$ and to zero otherwise (see Kaj10]).

Theorem 5.3. The ring homomorphisms

$$
\theta_{\mathrm{a}}: \mathbb{Q}[x, b] \rightarrow H_{K}^{*}\left(\mathrm{a} \Sigma^{\times}\right) \quad \text { and } \quad \theta_{\mathrm{w}}: \mathbb{Q}[x, b] \rightarrow H_{T_{\mathrm{w}}}^{*}(\mathrm{w} \Sigma)
$$

are surjective, where $\theta_{\mathrm{a}}:=\Pi^{*} \circ \theta$ and $\theta_{\mathrm{w}}:=\left(\Pi_{\mathrm{w}}^{*}\right)^{-1} \circ \theta_{\mathrm{a}}$. Here $\mathfrak{S}_{\sigma}$ is mapped to a $\tilde{\xi}_{\sigma^{-1}}$ by $\theta_{\mathrm{a}}$ and to $\mathrm{w} \tilde{\xi}_{\sigma^{-1}}$ by $\theta_{\mathrm{w}}$, if $\sigma \in W^{P}$ and to zero otherwise.

Proof. By composing the above map $\theta$ with isomorphisms in the commutative diagram (11), we get the required result.

By definition of double Schubert polynomial, we have

$$
\mathfrak{S}_{s_{d}}(x, b)=\mathfrak{S}_{s_{d}}(x) \mathfrak{S}_{\text {id }}(-b)+\mathfrak{S}_{\mathrm{id}}(x) \mathfrak{S}_{s_{d}}(-b) .
$$

Then by the standard properties of the Schubert polynomial $\mathfrak{S}(x)$,

$$
\mathfrak{S}_{s_{d}}(x, b)=x_{1}+\cdots+x_{d}-\left(b_{1}+\cdots+b_{d}\right) .
$$

We now define the double Schubert polynomial corresponding to $\tilde{\xi}_{\text {div }}$ as follows.

$$
\mathfrak{S}_{\text {div }}(x, b):=\sum_{i=1}^{r} \mathfrak{S}_{s_{d_{i}}}(x, b) .
$$

So we have $\mathfrak{S}_{\text {div }}(x, b)=x_{\text {id }}-b_{\text {id }}$, where

$x_{\mathrm{id}}:=r\left(x_{1}+\cdots+x_{d_{1}}\right)+(r-1)\left(x_{d_{1}+1}+\cdots+x_{d_{2}}\right)+\cdots+\left(x_{d_{r-1}+1}+\cdots+x_{d_{r}}\right)$. 
Corollary 5.4. The map

$$
\theta_{\mathrm{a}}: \mathbb{Q}[x, b] \rightarrow H_{K}^{*}\left(\mathrm{a} \Sigma^{\times}\right)
$$

is a homomorphism of algebras over $\mathbb{Q}\left[x_{\mathrm{id}}, b_{1}, b_{2}, \ldots\right]$.

Proof. As a consequence of the isomorphism $\Pi^{*}: H_{T}^{*}(\Sigma) \rightarrow H_{K}^{*}\left(\mathrm{a} \Sigma^{\times}\right)$and the definition (19) of $\tilde{\xi}_{\text {div }}$, we have

$$
\left(\Pi^{*}\right)^{-1}(z)=\tilde{\xi}_{\mathrm{div}}+y_{\mathrm{id}}
$$

This and the expression for $\mathfrak{S}_{\operatorname{div}}(x, b)$ give

$$
\theta_{\mathrm{a}}\left(x_{\mathrm{id}}\right)=\mathrm{a} \tilde{\xi}_{\mathrm{div}}+y_{\text {id }}=z .
$$

By Theorem 5.3, $\theta_{\mathrm{a}}$ is a homomorphism of algebras over $\mathbb{Q}[b]$. Thus we have extended the ring of coefficients to $\mathbb{Q}\left[x_{\mathrm{id}}, b_{1}, b_{2}, \ldots\right]$ by mapping $x_{\mathrm{id}}$ to $z, b_{i}$ onto $y_{i}$ for $1 \leq i \leq n$ and $b_{i}$ to zero for $i>n$.

Let $w_{l} \in \mathbb{Z}_{\geq 0}$, for $l \in \mathbb{N}$ be such that the first $n$ terms $w_{1}, \ldots, w_{n}$ are the same as weights chosen to define the weighted flag variety $\mathrm{w} \Sigma$. Let

$$
b_{l}^{\mathrm{w}}:=b_{l}+\frac{w_{l}}{u} x_{\mathrm{id}}, \quad l \in \mathbb{N} .
$$

As $\left\{b_{l}^{\mathrm{w}}: l \in \mathbb{N}\right\}$ is a set of algebraically independent variables so $\mathbb{Q}\left[b^{\mathrm{w}}\right]:=\mathbb{Q}\left[b_{1}^{\mathrm{w}}, b_{2}^{\mathrm{w}}, \ldots\right]$ is a polynomial ring. Moreover, we have a canonical isomorphism of rings

$$
\mathbb{Q}[x, b] \cong \mathbb{Q}\left[x, b^{\mathrm{w}}\right]
$$

via

$$
x_{i} \mapsto x_{i} \text { and } b_{l} \mapsto b_{l}^{\mathrm{w}}-\left(w_{l} / u\right) x_{\mathrm{id}}
$$

The following result follows from Corollary 5.4 .

Proposition 5.5. The map $\theta_{w}: \mathbb{Q}\left[x, b^{\mathrm{w}}\right] \rightarrow H_{T}^{*}(\mathrm{w} \Sigma)$ is a surjective homomorphism of algebras over $\mathbb{Q}\left[b^{\mathrm{w}}\right]$ via the following projection

$$
\mathbb{Q}\left[b^{\mathrm{w}}\right] \rightarrow \mathbb{Q}\left[y_{1}^{\mathrm{w}}, \ldots, y_{n}^{\mathrm{w}}\right],
$$

$b_{l}^{\mathrm{w}} \mapsto y_{l}^{\mathrm{w}}$ for $l=1, \ldots, n$ and $b_{l}^{\mathrm{w}} \mapsto 0$, otherwise.

Using the isomorphism of algebras $\mathbb{Q}[x, b] \cong \mathbb{Q}\left[x, b^{\mathrm{w}}\right]$ defined by $(22)$, we are now able to define weighted Schubert polynomials $\mathrm{wS}_{\sigma}\left(x, b^{\mathrm{w}}\right)$.

Definition 5.6. Weighted Schubert polynomials $\mathrm{wS}_{\sigma}\left(x, b^{\mathrm{w}}\right)$ are defined as images of double Schubert polynomials $\mathfrak{S}_{\sigma}(x, b)$ under the isomorphism $\mathbb{Q}[x, b] \cong \mathbb{Q}\left[x, b^{\mathrm{w}}\right]$. That is,

$$
\mathrm{w} \mathfrak{S}_{\sigma}\left(x, b^{\mathrm{w}}\right):=\sum_{\substack{\sigma=\tau^{-1} \mu \\ l(\sigma)=l(\tau)+l(\mu)}} \mathfrak{S}_{\tau}(x) \mathfrak{S}_{\mu}\left(b_{1}^{\mathrm{w}}-\left(w_{1} / u\right) x_{\mathrm{id}}, b_{2}^{\mathrm{w}}-\left(w_{2} / u\right) x_{\mathrm{id}}, \ldots\right) .
$$

We give a description of weighted Schubert classes in terms of weighted Schubert polynomials. We will use the following result of Kaji on double Schubert polynomials. 
Proposition 5.7. (Kaj11, Prop. 4.9]) For $\sigma, \tau$ in $W^{P}$, we have

$$
\mathfrak{S}_{\sigma}\left(b_{\tau(1)}, \ldots, b_{\tau(n)} ; b\right)=\left\{\begin{array}{cc}
\prod_{(i, j) \in \operatorname{Inv}_{P}(\sigma)}\left(b_{j}-b_{i}\right) & \tau=\sigma \\
0 & \tau \nsucceq \sigma
\end{array} .\right.
$$

In particular, for $b_{i}=y_{i}, \forall i \in \mathbb{N}$, where $y_{i}=0$ for all $i>n$, we have

$$
\mathfrak{S}_{\sigma}\left(b_{\tau(1)}, \ldots, b_{\tau(n)} ; b\right)=\left.\tilde{\xi}_{\sigma}\right|_{\tau} .
$$

Consider the map $\alpha_{\tau}: \mathbb{Q}\left[x, b^{\mathrm{w}}\right] \rightarrow \mathbb{Q}\left[b^{\mathrm{w}}\right]$ defined as

$$
x_{i} \mapsto b_{\tau_{i}}^{\mathrm{w}}-\left(w_{\tau_{i}} / w_{\tau}\right) b_{\tau}^{\mathrm{w}} \forall i=1, \ldots, n,
$$

where $\tau_{i}=\tau(i)$. The image of $x_{\mathrm{id}}$ under the map $\alpha_{\tau}$ is $\left(u / w_{\tau}\right) b_{\tau}^{\mathrm{w}}$. Thus we have:

$$
\alpha_{\tau}\left(\mathrm{w}_{\sigma}\left(x, b^{\mathrm{w}}\right)\right)=\mathfrak{S}_{\sigma}\left(d_{\tau_{1}}^{\mathrm{w}}, \ldots, d_{\tau_{n}}^{\mathrm{w}}, d_{1}^{\mathrm{w}}, d_{2}^{\mathrm{w}}, \ldots\right)
$$

for $\sigma, \tau \in W^{P}$, where $d_{\tau_{i}}^{\mathrm{w}}=b_{\tau_{i}}^{\mathrm{w}}-\left(w_{\tau_{i}} / w_{\tau}\right) b_{\tau}^{\mathrm{w}}$.

Lemma 5.8. For $\sigma, \tau$ in $W^{P}$, we have

$$
\mathrm{w} \tilde{\xi}_{\sigma}\left|\tau=\alpha_{\tau}\left(\mathrm{w}_{\sigma}\left(x, b^{\mathrm{w}}\right)\right)\right|_{b_{i}^{\mathrm{w}}=y_{i}^{\mathrm{w}}} .
$$

Proof. The previous Proposition and 24 give us the desired expression for $\mathrm{w} \tilde{\xi}_{\sigma} \mid \tau$.

We define the weighted Schubert polynomial $\mathrm{w}_{\sigma}(x)$ as

$$
{ }_{\mathrm{w}} \mathfrak{S}_{\sigma}(x, 0)=\mathfrak{S}_{\sigma}\left(x,-\left(w_{1} / u\right) x_{\mathrm{id}}, \ldots\right) .
$$

Proposition 5.9. The polynomials $\mathrm{w}_{\sigma}(x), \sigma \in W^{P}$ form a $\mathbb{Q}$-basis of $\mathbb{Q}\left[x_{1}, \ldots, x_{n}\right]^{W_{P}} / I$, where $I$ is the ideal generated by

$$
f\left(x_{1}, \ldots, x_{r}\right)-f\left(\frac{-w_{i}}{u} x_{\mathrm{id}}, \ldots, \frac{-w_{r}}{u} x_{\mathrm{id}}\right)
$$

for all $W$-invariant polynomials $f$ of positive degree. Moreover, there is a surjective ring homomorphism

defined as

$$
\frac{\mathbb{Q}\left[x_{1}, \ldots, x_{n}\right]^{W_{P}}}{I} \rightarrow H^{*}(\mathrm{w} \Sigma)
$$

$$
\mathrm{w} \mathfrak{S}_{\sigma}(x) \mapsto \mathrm{w} \xi_{\sigma^{-1}}
$$

Proof. By Borel Construction [GHZ06, p.24], it is well-known that

$$
H_{T}^{*}(\Sigma) \cong \frac{\mathbb{Q}\left[x_{1}, \ldots, x_{n}\right]^{W_{P}} \otimes \mathbb{Q}\left[b_{1}, \ldots, b_{n}\right]}{I},
$$

where $I$ is the ideal generated by $f\left(x_{1}, \ldots, x_{r}\right)-f\left(b_{1}, \ldots, b_{r}\right)$ for all $W$-invariant polynomials $f$ of positive degree. Also, the double Schubert polynomials $\left\{\mathfrak{S}_{\sigma}(x, b): \sigma \in W_{P}\right\}$ form a basis for

$$
\frac{\mathbb{Q}\left[x_{1}, \ldots, x_{n}\right]^{W_{P}} \otimes \mathbb{Q}\left[b_{1}, \ldots, b_{n}\right]}{I} .
$$

Thus the result follows from the definition of weighted Schubert polynomial. 
Theorem 5.10. For any simple reflection $s_{d} \in W^{P}$ and $\sigma \in W^{P}$, the Chevalley-Monk's formula for weighted double Schubert polynomials is given by

$$
\mathrm{w}_{S_{d}}(x) \mathrm{w} \mathfrak{S}_{\sigma}(x)=\frac{\sum_{i=1}^{d}\left(w_{i}-w_{\sigma_{i}}\right)}{u} x_{\mathrm{id}} \mathrm{w} \mathfrak{S}_{\sigma}(x)+\sum_{\substack{i \leq d<j \\ l((i j) \sigma)=l(\sigma)+1}} \mathrm{w} \mathfrak{S}_{(i j) \sigma}(x) .
$$

Proof. From[Man01], we have a nice formula for the product of double Schubert polynomials

$$
\mathfrak{S}_{s_{d}}(x, b) \mathfrak{S}_{\sigma}(x, b)=\left(b_{\sigma_{1}}+\cdots+b_{\sigma_{d}}-b_{1}-\cdots-b_{d}\right) \mathfrak{S}_{\sigma}(x, b)+\sum_{\substack{i \leq d<j \\ l((i j) \sigma)=l(\sigma)+1}} \mathfrak{S}_{(i j) \sigma}(x, b)
$$

Replacing $b_{i}$ by $-\frac{w_{i}}{u} x_{\mathrm{id}}$ in above equation, we get the result.

As a concluding remark, it is opportune to mention that the geometric and topological aspects (including Schubert calculus) of weighted flag varieties, as a subject is out there to be explored. We expect this to grow into an area with potential research directions, for instance ANQ. The next obvious question seems to be of computing cohomology of complete intersections in weighted flag orbifolds. Similarly we hope that results from equivariant $K$-theory and equivariant quantum cohomology of homogeneous spaces could also be generalized to these spaces.

Acknowledgement. We wish to thank Waqar Ali Shah for several helpful discussions. We also thank Frank Sottile and Balázs Szendrői for their comments on earlier drafts of this article. Thanks are also due to Shizu Kaji, Allen Knutson and Loring Tu for some useful conversations. Last but not least, we are indebted to the anonymous referee for his/her comments and suggestions which significantly improved the earlier version of this paper. HA and MIQ were supported by the HEC's NRPU research grant "5906/Punjab/NRPU/HEC/2016". MIQ was on a fellowship of Alexander-Von-Humboldt foundation during a part of this paper.

\section{REFERENCES}

[AB94] MF Atiyah and R Bott, The moment map and equivariant cohomology, Collected Papers (1994), 241.

[Alb86] Arabia Alberto, Cohomologie T-equivariante de $G / B$ pour an groupe $G$ de kac-moody, C.R.Acad.Sci.Paris 302 (1986), 631-634.

[AM15a] Hiraku Abe and Tomoo Matsumura, Equivariant cohomology of weighted Grassmannians and weighted Schubert classes, Int. Math. Res. Not. IMRN (2015), no. 9, 2499-2524. MR 3344679

[AM15b] Schur polynomials and weighted Grassmannians, Journal of Algebraic Combinatorics 42 (2015), no. 3, 875-892.

[ANQ] Haniya Azam, Shaheen Nazir, and Muhammad Imran Qureshi, Schubert calculus on weighted symplectic Grassmanians, In progress.

$\left[\mathrm{BBF}^{+} 60\right]$ Armand Borel, G. Bredon, E. E. Floyd, D. Montgomery, and R. Palais, Seminar on transformation groups, vol. (46), Princeton University Press, Princeton, 1960.

[BKZ14] Gavin Brown, Alexander Kasprzyk, and Lei Zhu, Gorenstein formats, canonical and Calabi-Yau threefolds, arXiv preprint arXiv:1409.4644 (2014). 
$\left[\mathrm{BM}^{+} 60\right]$ Armand Borel, James C Moore, et al., Homology theory for locally compact spaces., The Michigan Mathematical Journal 7 (1960), no. 2, 137-159.

[Bre72] Glen E Bredon, Introduction to compact transformation groups, vol. 46, Academic press, 1972.

[Bri05] Michel Brion, Lectures on the geometry of flag varieties, Topics in cohomological studies of algebraic varieties, Springer, 2005, pp. 33-85.

$\left[\mathrm{BV}^{+} 83\right]$ Nicole Berline, Michele Vergne, et al., Zéros dun champ de vecteurs et classes caractéristiques équivariantes, Duke Mathematical Journal 50 (1983), no. 2, 539-549.

[CLS11] David A Cox, John B Little, and Henry K Schenck, Toric varieties, American Mathematical Soc., 2011.

[CR02] Alessio Corti and Miles Reid, Weighted Grassmannians, Algebraic geometry, de Gruyter, Berlin, 2002, pp. 141-163. MR 1954062

[CS73] Theodore Chang and Tor Skjelbred, Topological schur lemma and related results, Bulletin of the American Mathematical Society 79 (1973), no. 5, 1036-1038.

[Ehr34] Charles Ehresmann, Sur la topologie de certains espaces homogenes, Annals of Mathematics 35 (1934), no. 2, 396-443.

[FH13] William Fulton and Joe Harris, Representation theory: a first course, vol. 129, Springer Science \& Business Media, 2013.

[Ful97] William Fulton, Young tableaux: with applications to representation theory and geometry, vol. 35, Cambridge University Press, 1997.

[GHZ06] Victor Guillemin, Tara Holm, and Catalin Zara, A GKM description of the equivariant cohomology ring of a homogeneous space, Journal of Algebraic Combinatorics 23 (2006), no. 1, 21-41.

[GKM97] Mark Goresky, Robert Kottwitz, and Robert MacPherson, Equivariant cohomology, koszul duality, and the localization theorem, Inventiones mathematicae 131 (1997), no. 1, 25-83.

[Kaj10] Shizuo Kaji, Schubert calculus, seen from torus equivariant topology, Trends in MathematicsNew Series 12 (2010), no. 1, 71-90.

[Kaj11]__ Equivariant schubert calculus of Coxeter groups, Proceedings of the Steklov Institute of Mathematics 275 (2011), no. 1, 239-250.

[Kir84] Frances Clare Kirwan, Cohomology of quotients in symplectic and algebraic geometry, Mathematical Notes, vol. 31, Princeton University Press, Princeton, NJ, 1984. MR 766741

[KK86] Bertram Kostant and Shrawan Kumar, The nil Hecke ring and cohomology of gp for a kac-moody group g, Advances in Mathematics 62 (1986), no. 3, 187-237.

[Man01] Laurent Manivel, Symmetric functions, schubert polynomials, and degeneracy loci, no. 3, American Mathematical Soc., 2001.

[QS11] M. I. Qureshi and B. Szendrői, Constructing projective varieties in weighted flag varieties, Bull. Lon. Math Soc. 43 (2011), no. 2, 786-798.

[QS12] M. I. Qureshi and Balázs Szendrői, Calabi-Yau threefolds in weighted flag varieties, Adv. High Energy Phys. (2012), Art. ID 547317, 14 pp.

[Qur15] M. I. Qureshi, Constructing projective varieties in weighted flag varieties II, Math. Proc. Camb. Phil. Soc. 158 (2015), 193-209.

[Qur17a] Muhammad Imran Qureshi, Computing isolated orbifolds in weighted flag varieties, Journal of Symbolic Computation 79, Part 2 (2017), 457 - 474.

[Qur17b] _ Polarized 3-folds in a codimension 10 weighted homogeneous F-4 variety, Journal of Geometry and Physics 120 (2017), 52 - 61.

[Tym09] Julianna S Tymoczko, Divided difference operators for partial flag varieties, arXiv preprint arXiv:0912.2545 (2009).

Haniya Azam, Department of Mathematics, SBASSE, Lahore University of Management SCIENCES (LUMS), LAhore, PAKISTAN

E-mail address: haniya.azam@lums.edu.pk 
Shaheen Nazir, Department of Mathematics, SBASSE, Lahore University of Management Sciences (LUMS), LAhore, PAKistan

E-mail address: shaheen.nazir@lums.edu.pk

Muhammad Imran Qureshi, Department of Mathematics, SBASSE Lahore University of Management Sciences (LUMS), Lahore, Pakistan and Mathematisches Institut, Universität TüBIngen, GERMANy

E-mail address: i.qureshi@maths.oxon.org 